\title{
Una propuesta para impulsar el espíritu emprendedor y la capacitación en el futuro de la profesión periodística
}

\author{
Pedro Aceituno-Aceituno \\ Universidad a Distancia de Madrid \\ pedro.aceituno@udima.es \\ Carlos Bousoño CALzÓN \\ Universidad Carlos III de Madrid \\ cbousono@tsc.uc3m.es \\ Francisco José HeRrera GÁlvez \\ Universidad Carlos III de Madrid \\ fherrera54@gmail.com
}

Recibido: 11 de marzo de 2014

Aceptado: 29 de septiembre de 2014

\section{Resumen}

La profesión periodística puede ofrecer una prestigiosa carrera a sus futuros graduados, pero la situación del sector es crítica y llena de cambios a los que nuevos periodistas deber responder con una mayor capacitación profesional, que les permita ofrecer una mayor calidad en el servicio al consumidor. Ante este escenario, la integración del emprendimiento en la formación de los periodistas resulta clave para que la innovación y la creatividad impulsen el autoempleo o nuevas iniciativas en las empresas periodísticas existentes. Para que esta formación sea más efectiva, en este estudio se expone la experiencia de una propuesta docente que emplea una metodología de aprendizaje de servicio, cuyos resultados corroboran su importancia didáctica para fomentar el espíritu emprendedor y la capacitación de los futuros periodistas.

Palabras clave: Periodistas, emprendimiento, formación, calidad, carrera.

\section{A proposal to encourage entrepreneurship and training in the future of journalism}

\begin{abstract}
Journalism can offer a prestigious career development to their future graduates, but the current situation for this activity sector is critical and full of changes the new journalists must respond to. This response has to be based upon a better professional training able to provide a higher quality customer service. Considering this scenario, the integration of entrepreneurship in the journalist's education is key to make innovation and creativity boost self-employment and new initiatives in existing media companies. In order to contribute towards a more effective training in this area, a teaching proposal based on the methodology of service learning is shown. Our results support the importance of education to encourage entrepreneurship and its associated skills in future journalists.
\end{abstract}

Keywords: Journalists, entrepreneurship, training, quality, career.

\section{Referencia normalizada}

ACEITUNO-ACEITUNO, Pedro; BOUSOÑO-CALZÓN, Carlos; y HERRERA GÁLVEZ, Francisco José (2015): "Una propuesta para impulsar el espíritu emprendedor y la capacitación en el futuro de la profesión periodística". Estudios sobre el Mensaje Periodístico. Vol. 21, Núm. 2 (julio-diciembre), págs.: 929-942. Madrid, Servicio de Publicaciones de la Universidad Complutense. 
Sumario: 1. Importancia y necesidad del periodismo emprendedor en la creación y desarrollo de nuevos modelos de negocio basados en la calidad. 2. Una metodología docente para ofrecer servicios a la sociedad, las empresas y los emprendedores; 2.1. Contenido de la propuesta docente principal; 2.2. Ajuste a la realidad social y empresarial de la propuesta docente; 2.3 . Servicios ofrecidos a la sociedad y a las empresas y emprendedores. 3. Resultados. 4. Conclusiones. 5. Referencias bibliográficas.

\section{Importancia y necesidad del periodismo emprendedor en la creación y des- arrollo de nuevos modelos de negocio basados en la calidad ${ }^{1}$}

A finales de la década pasada Larrañaga-Rubio (2009: 63) destacaba la disminución en la venta de periódicos impresos en la mayoría de los países de Europa Occidental, Norteamérica y Oceanía y las pesimistas previsiones a nivel mundial en la cifra de negocio de esta industria para los próximos años. Estas últimas se han cumplido con una reducción de los ingresos por ventas y publicidad (127.172 millones de euros en 2012 frente a 145.803 del año 2008), aunque se espera que tras un periodo de descenso en el volumen de facturación, este se estabilice en los niveles de 2012 hasta el año 2017, debido a que la sostenida expansión de los mercados emergentes (Rusia, India, Brasil, China, Argentina, Oriente Próximo, Norte de Africa, Indonesia y México) compensará el declive continuo en los mercados maduros como Europa Occidental y Central o Norteamérica (PricewaterhouseCoopers, 2013: 16 y 33).

Para garantizar su supervivencia, las empresas periodísticas están buscando nuevos modelos de negocio (Larrañaga-Rubio, 2010: 75), en los que los medios deben afrontar no sólo la competencia entre ellos, sino también la que les ofrecen los nuevos medios digitales, que rivalizan también por los ingresos publicitarios y utilizan nuevos modelos basados en la cooperación y participación de los usuarios (Campos, 2010: 19). Por ello, la refundación de los modelos de negocio de la prensa para adaptarse a la convergencia digital es una necesidad imperiosa (Casero, 2010: 596). En este sentido, las tecnologías digitales también suponen nuevas opciones y características técnicas para la generación de contenidos y de nuevos modelos de negocio (Casero, 2012: 342).

De forma general, la innovación en modelos de negocio consiste en desafiar las normas para generar modelos originales que satisfagan todas las necesidades de los clientes: desatendidas, nuevas u ocultas (Osterwalder y Pigneur, 2011: 136). Si esto se aplica en referencia a los generadores y distribuidores de contenidos, los nuevos modelos de negocio han de estar orientados a conocer y entender los hábitos compradores y de expectativas del nuevo consumidor digital. En ese sentido, es necesario destacar que este nuevo consumidor se ha sofisticado, busca contenidos más personalizados y relevantes, a través de diferentes dispositivos o plataformas, y en el momento y con el precio adecuado (PricewaterhouseCoopers, 2013: 3).

1 Los autores de este trabajo quieren agradecer a todos los empresarios, emprendedores y alumnos que han posibilitado que pueda realizarse esta propuesta docente, y muy especialmente a los futuros periodistas emprendedores de la Universidad a Distancia de Madrid (UDIMA). También, se agradece el patrocinio de este trabajo por Novanca. Igualmente se muestran agradecimientos a la UDIMA por todos los recursos económicos, materiales y humanos dispuestos para efectuar este estudio mediante su Programa de Creación y Consolidación de Grupos de Investigación (referencia UI2011-2). 
Dentro de estos nuevos modelos, la apuesta por un periodismo que sea capaz de proporcionar contenidos de alta calidad se ofrece como la mejor solución para mantener estos signos de esperanza para la industria de la prensa mundial, que como se expuso anteriormente, no eclipsan las dificultades muy reales que se padecen en otras partes, particularmente en los países occidentales (Thompson, 2013: 29). Igualmente, Meyer (1995: web) destaca estos aspectos cualitativos a los propietarios de los periódicos, cuando les avisa de que deben ajustarse a las nuevas realidades de un negocio con márgenes de beneficios más reducidos y en los que hay que tratar de cultivar los productos para generar mejores contenidos que los de la competencia.

Además, a este difícil escenario se une que los mayores cambios que se han producido en la profesión periodística en los últimos 100 años, han tenido lugar a comienzos del siglo XXI, siendo los retos para afrontarlos muy grandes tanto en la propia industria como en la enseñanza del periodismo (Flew, 2012: 2). Precisamente, dentro de este contexto de crisis del sector, cambios en el entorno comunicativo y tecnologías digitales que reducen las barreras de entrada para impulsar nuevos proyectos periodísticos, Casero-Ripollés y Cullel-March (2013: 686) afirman que resulta básico integrar el emprendimiento como una herramienta fundamental en la formación de los periodistas, para que la innovación y la creatividad propicien la aparición de un autoempleo que genere nuevas iniciativas y servicios periodísticos.

El hecho de que los periodistas puedan dedicar esta formación al autoempleo, también puede ser aprovechado en beneficio de la innovación y la creatividad de las empresas periodísticas existentes, pues como afirma Goñi (2013: 35), las personas con estas capacidades son personas que presentan los siguientes rasgos:

- Creativas en la identificación de nuevas soluciones y oportunidades.

- Con actitud positiva ante el cambio y la innovación.

- Equilibradas emocionalmente para abordar situaciones difíciles, controlar sus emociones, superar las derrotas y críticas o implantar nuevas soluciones.

- Capaces de aprender de sus errores y sin miedo al error.

- Preparados para idear, desarrollar e implementar proyectos y todo lo que conlleva su puesta en marcha: liderazgo, planificación, organización, comunicación, evaluación y aprendizaje.

Dada la importancia que puede tener que los futuros periodistas, puedan conocer los modelos de negocios desde el interior de las compañías, y con este aprendizaje proyectar con mayores elementos de juicio nuevas iniciativas dentro del periodismo, en este trabajo se amplia el concepto de emprendedor desde el autoempleo a todos aquellos periodistas capaces de generar, implantar y desarrollar proyectos en cualquier ámbito de actuación de una organización del sector, siguiendo en este sentido la definición que proporciona la Real Academia Española de la Lengua (RAE) para el concepto de emprender, cuyo significado es el de "acometer y comenzar una obra, un negocio, un empeño, especialmente si encierra dificultad o peligro".

De forma simultánea a las transformaciones económicas que se iban produciendo en el último tercio del siglo XX, las administraciones públicas y la universidad han ido prestando un mayor interés al fenómeno del emprendimiento (Ortega, 2012: 71). Dado 
el actual contexto de crisis y de globalización, esta tendencia creciente de apoyo institucional público, privado y universitario debe continuar, ya que se hace muy necesaria la presencia de personas con una elevada cultura emprendedora, que sean capaces de dinamizar el sistema socio-económico (Jiménez y Legato, 2010: 7).

En este sentido, aunque los valores culturales de cada país puedan influir en la forma de estimular las intenciones emprendedoras de cada persona (Liñán y Chen, 2009: 610), la educación en emprendimiento es un instrumento básico para que estas intenciones puedan forjarse y desarrollarse (Peterman y Kennedy, 2003: 141). El empresario se hace, no nace, y resulta necesario que se le capacite, de tal manera que pueda aprovechar las oportunidades que le surjan (Krueger y Brazeal, 1994: 102). No obstante, los efectos de esta formación no son muy conocidos ni consistentes, por lo que se hace preciso estudiar los tipos de procesos de aprendizaje que facilitan estos beneficiosos efectos sobre el emprendimiento (Von Graevenitz et al., 2007: 103-104).

El periodismo es una profesión fascinante que todavía ofrece una emocionante carrera a muchos jóvenes brillantes, pero existe el peligro de que algunos graduados, que se incorporan a la profesión con las mejores habilidades e intenciones, puedan ser los primeros desencantados y estén tentados a abandonar la misma (Edwards, 2013: 334). Precisamente, lo crítico de la situación provoca que aparezcan nuevos retos y oportunidades, de las que deberían originarse nuevas experiencias y formas de periodismo (Guallar, 2010: 165), que hay que saber aflorar para que los futuros periodistas se sientan protagonistas del destino de su profesión, quieran defenderla, encuentren nuevas formas de enriquecerla y entiendan que son pieza básica para ayudar a muchas personas e instituciones dentro de la sociedad.

Por todo lo expuesto anteriormente, resulta conveniente aportar procesos o propuestas a la educación en periodismo que tengan en cuenta estos aspectos. Para ello, a continuación se expone una propuesta que por un lado, trata de estimular el espíritu emprendedor y por el otro, intenta motivar al alumno en su capacitación para que pueda ofrecer una mayor calidad en su manera de proceder profesionalmente, tanto en los contenidos que genere como en su manera de conocer al consumidor y mantener su atención. Todo ello, además amplia el conocimiento empírico sobre la creación y gestión de los medios de comunicación, en general, y sobre la empresa periodística en particular, como ciencia naciente, que abarca múltiples disciplinas (Campos, 2010: $15)$.

\section{Una metodología docente para ofrecer servicios a la sociedad, las empresas y los emprendedores}

La metodología docente utilizada en la propuesta educativa que se plantea en el presente estudio es la de aprendizaje de servicio, debido a que como han puesto de manifiesto Casero-Ripollés y Cullel-March (2013: 687) es una metodología que contribuye con éxito a formar a los periodistas emprendedores del futuro. Según Martínez (2008: 21), esta metodología propone un trabajo cooperativo y/o colaborativo que ayude a que el estudiante tome conciencia de su capacidad para conseguir una transformación social mediante el desarrollo de competencias que le aproximen a la realidad medioambiental, personal, económica, social o cultural de una colectividad. 
Igualmente, se sigue a Jones (2005: 34) para analizar con unos mayores elementos de juicio esta propuesta formativa cuando expone que no son excesivos los estudios académicos y profesionales sobre las industrias comunicativas de componente transversal, es decir, de carácter comparativo entre empresas, industrias o colectivos profesionales, entre otros. En concreto, en el caso que se expone en este estudio, se trata de conocer el nivel de adecuación de la propuesta educativa sobre el impulso del espíritu emprendedor y la capacitación de los futuros graduados en Periodismo comparando dicho nivel con el que muestran otros futuros graduados de otras profesiones.

La utilización de esta metodología resulta aconsejable debido a que permite que el estudiante pueda aproximarse a la realidad con la que tendrá que interactuar (empresas, emprendedores, clientes o trabajadores, por ejemplo), favorece la toma de conciencia del alumnado de que una de las vías para recuperar en la prensa valores como la credibilidad, la reputación y la confianza pasa porque se implante en su gestión la Responsabilidad Social Empresarial (RSE) como una práctica sistemática verificable (Campos, 2010: 25-27), lo cual se puede hacer extensible al resto de sectores de la economía y a sus futuros graduados, y por último, ayuda a que el alumno a la vez que está estudiando pueda aplicar sus conocimientos.

En el caso de los graduados de periodismo, resulta igualmente adecuado que este estudio se efectúe desde una universidad española como es la Universidad a Distancia de Madrid (UDIMA), dado que las cifras del Informe de la Profesión Periodística 2012 (Palacios-Llanos, 2012: 27), ponen de manifiesto que prácticamente el 80 por 100 de los jóvenes periodistas españoles no tienen ninguna esperanza o consideran que será muy difícil encontrar algún trabajo relacionado con el periodismo durante los próximos 12 meses.

Todo ello, hace necesario que los futuros graduados en periodismo estén fuertemente impregnados por los valores del emprendimiento y también por los deseos de capacitarse para que puedan ayudar a cambiar y a enriquecer los modelos de negocio que faciliten que el sector pueda superar esta situación tan crítica. Para cumplir con estos objetivos, la metodología docente utilizada en este estudio se fundamenta en los tres elementos, que se exponen a continuación.

\subsection{Contenido de la propuesta docente principal}

Las dos aulas de la UDIMA en las que se ha aplicado esta metodología docente han sido las de Creación y gestión de empresas e Introducción a la organización de empresas durante los cursos académicos 2012-2013 y 2013-2014. La primera de estas aulas tiene un claro carácter emprendedor y en la segunda, de componente más introductoria en el estudio de la empresa, se ha fomentado esta competencia mediante la realización de la propia propuesta, la utilización de una red social interna para exponer noticias de emprendimiento, sesiones de videoconferencia o emisión de vídeos que cuentan diversas experiencias de empresarios y emprendedores.

Por las razones expuestas anteriormente, esta metodología de servicio ha sido utilizada tanto en aulas de estudiantes de Periodismo como de otros grados. Los grados a los que pertenecen el resto de alumnos son los de Administración y dirección de empresas, Derecho y Ciencias del trabajo y recursos humanos para el aula de Creación 
y gestión de empresas, y estos mismos alumnos junto a los de Empresas y actividades turísticas, Ingeniería informática, Psicología y Magisterio en educación primaria para el aula de Introducción a la organización de empresas.

El contenido básico de esta propuesta docente principal en cada una de las aulas ha sido el siguiente:

- Aulas de Creación y gestión de empresas de Periodismo:

- Curso 2012-2013-Primer semestre: los alumnos han tenido que realizar una propuesta de modelo de negocio con la vocación social de generar empleo. Con ello, han aplicado los conocimientos obtenidos para tratar de transformar la difícil realidad del colectivo de desempleados. De esta manera, se trata de que entiendan el emprendimiento como un proyecto de construcción personal con el que se puede favorecer a otras personas.

- Curso 2013-2014-Primer semestre: De acuerdo con García-López y MartínezPastor (2013: 177) se destaca la conveniencia de integrar el conocimiento de las redes sociales en las enseñanzas, por lo que en esta actividad didáctica los estudiantes han ayudado a dos emprendedores en la realización de sus planes de gestión de redes sociales. Estos proyectos han sido el de la empresa de desarrollo de software Seven peaks, dedicada a facilitar las tareas diarias de sus clientes y la creación de una escuela de educación infantil. La estructura del contenido de estos planes ha sido abierta, pero se ha recomendado que en la misma aparezcan algunos aspectos como los siguientes:

- Objetivos del plan.

- Medidas a tomar para cumplir estos objetivos:

- Mecanismos para observar lo publicado en materias que le puedan interesar al proyecto empresarial.

- Formas para hacer accesible esta información al emprendedor.

- Comunicar la posición de la empresa en relación con temas de su interés y de la zona principal de su actuación.

- Promocionar la empresa en las redes sociales.

- Localizar y conectar con el emprendedor a personas que pueden estar interesadas en la marcha de la empresa (clientes, proveedores, financiadores, entre otros).

- Resultados probables (volumen general desglosado de ingresos y costes asociados a la puesta en marcha de las medidas para cumplir los objetivos).

- Aula de Creación y gestión de empresas de otros grados (Curso 2012-2013-Segundo semestre): los alumnos han apoyado a cuatro emprendedores con la formulación de una propuesta de modelo de negocio para las ideas planteadas por ellos. Estos proyectos han sido los siguientes: creación de un sistema de información turística, generación de un desarrollo de software que analice los sentimientos sobre marcas en internet, establecimiento de una plataforma de gestión de eventos culturales en Madrid y desarrollo de la publicación Villaviciosa digital. Además, de esta propuesta los alumnos han valorado los proyectos de los emprendedores mediante una herramienta de inteligencia colectiva denominada mercados de información (MI), que ha sido empleada en el ámbito laboral de 
grandes empresas, como Google o Pfizer, para incentivar la creatividad y el compromiso de los trabajadores (Thompson, 2013: 83 y 239).

- Aulas de Introducción a la organización de empresas:

- Curso 2012-2013-Segundo semestre: los alumnos han aplicado los conocimientos adquiridos al diseño de nuevas estructuras organizativas o a la realización de planes de comercialización, recursos humanos o internacionalización de los proyectos que empresarios y emprendedores les han propuesto. Los proyectos apoyados han sido los siguientes: una gestoría administrativo-económica (De IURE international consulting), una agencia de viajes dedicada al mundo del golf (Birdie tours), un proyecto de creación de un Skatepark indoor en Madrid, una agencia de organización de eventos musicales en el exterior (Networking crescendo), una gestoría administrativoeconómica (Asesoría integral de empresas) y Seven peaks, empresa citada anteriormente. Igualmente, los alumnos han valorado los proyectos de los empresarios y emprendedores mediante los MI.

- Curso 2013-2014-Primer semestre: los estudiantes han ayudado a 22 compañeros del propio aula, que quieren ser emprendedores, realizando idénticas actividades a las expuestas en el semestre precedente.

\subsection{Ajuste a la realidad social y empresarial de la propuesta docente}

Con este tipo de ajuste se pretende que los alumnos puedan desarrollar la competencia de emprendimiento, acercándose a las diferentes realidades de los proyectos solicitados. Marina (2010: 59) expone que esta competencia es muy ambiciosa, ya que en gran medida de ella depende el futuro vital y profesional del alumno, al englobarse en la misma cualidades intelectuales (capacidad para realizar proyectos, planificar y evaluar de forma crítica), morales (perseverancia, valentía y responsabilidad) y sociales (cooperación, trabajo en equipo y liderazgo).

En el caso del aula Creación y gestión de empresas de Periodismo (Curso 20122013-Primer semestre), el hecho de que la propuesta consista en la realización de un proyecto generador de puestos de trabajo, trata de desarrollar todas estas cualidades, pero muy especialmente las del liderazgo y responsabilidad, ya que propone al alumno convertirse en el líder de un proyecto, con el que se puede cambiar el destino de las personas que están en la difícil situación del desempleo.

Igualmente, diversos estudios efectuados a nivel nacional (Toledano-Garrido, 2006:822; Fernández-Laviada et al., 2011: 19-20) aconsejan facilitar al alumno el contacto con empresarios y emprendedores para fomentar el desarrollo del espíritu emprendedor. Siguiendo el procedimiento propuesto por estos autores, los estudiantes del resto de aulas, han tenido la posibilidad de desarrollar cualidades emprendedoras a través de su cooperación con los emprendedores y empresarios expuestos anteriormente, que les han proporcionado sus ideas para que ellos las conviertan en proyectos que se puedan llevar hacia delante con un mayor grado de factibilidad.

En todos estos casos, se han habilitado dentro de las aulas virtuales foros para que todos los componentes del aula y los empresarios y emprendedores, pudieran cooperar e interactuar. Con ello, igualmente se ha pretendido motivar al alumno para que 
presente y contraste sus ideas con los destinatarios de su trabajo, lo que puede enriquecer cualitativamente los proyectos finalmente obtenidos.

\subsection{Servicios ofrecidos a la sociedad y a las empresas y emprendedores}

Con la puesta en marcha de esta propuesta docente se ha tratado de desarrollar esta actitud de servicio en el alumnado para que tuviera los siguientes efectos sobre la sociedad y las empresas y emprendedores:

- La generación de un pensamiento creativo en los alumnos que concibe al emprendimiento como un instrumento transformador de la realidad social de muchas personas, que están desempleadas.

- La proposición de nuevos planes a empresas y emprendedores para mejorar sus proyectos iniciales y que los mismos puedan alcanzar un mayor grado de viabilidad.

- La valoración colectiva y competitiva realizada por los alumnos mediante los MI facilita a los empresarios y emprendedores una primera percepción del valor que tienen sus proyectos, puede estimular sus intenciones emprendedoras ante el reconocimiento de potenciales clientes y sirve también de impulso para la mejora de los citados proyectos.

\section{Resultados}

Para valorar los efectos de esta propuesta docente se ha realizado una encuesta entre una muestra de 196 alumnos de los distintos grados en los que se ha realizado la misma. En el tabla 1 se puede observar el número de estudiantes de cada grado y su porcentaje dentro del total de la muestra, figurando los estudiantes de Periodismo en cuarto lugar.

Tras recibir la formación completa, los alumnos fueron consultados por su disponibilidad a emprender, mostrándose también en la tabla 1 el número de alumnos que han contestado afirmativamente y su porcentaje con respecto al parcial de cada grado. A la vista igualmente de la citada tabla 1, para unos porcentajes elevados de disponibilidad al emprendimiento en la mayoría de los grados, son los estudiantes del grado de Periodismo los que figuran en primera posición, superando a alumnos de titulaciones más relacionadas con el mundo de la empresa, por lo que se puede afirmar que la formación en emprendimiento recibida ha supuesto una referencia importante que ha impulsado su espíritu emprendedor.

Para profundizar en lo que ha supuesto la actividad principal en este fomento del emprendimiento, los estudiantes respondieron a la pregunta que figura en la tabla 2, con una escala de Likert de siete puntos, cuyos valores bajos, expresan una actividad didáctica muy adecuada, y los valores altos, una actividad didáctica nada adecuada. En base a los datos de esta tabla, los resultados del análisis de la varianza (ANOVA) revelan que existen diferencias estadísticamente significativas en la media de los valores de la pregunta entre el grado de periodismo y cada uno de los grados, respectivamente.

A la vista de los datos de la tabla 2, se puede afirmar que la propuesta docente resulta adecuada para fomentar el espíritu emprendedor de los estudiantes, pues la mayoría han 
respondido con valores bajos, a excepción de los alumnos de los grados de Psicología y de Empresas y actividades turísticas, que han presentado valores más neutros.

Tabla 1. Número y porcentaje de alumnos por grado y disponibilidad a emprender

\begin{tabular}{|c|c|c|c|c|}
\hline \multirow[b]{2}{*}{ Grado } & \multirow[b]{2}{*}{$\begin{array}{l}\text { Núm. de } \\
\text { alumnos }\end{array}$} & \multirow[b]{2}{*}{$\%$} & \multicolumn{2}{|c|}{ ¿Piensas que alguna vez emprenderás? } \\
\hline & & & $\begin{array}{l}\mathrm{N}^{0} \text { de alumnos } \\
\text { que han } \\
\text { respondido } \\
\text { afirmativamente }\end{array}$ & $\begin{array}{c}\text { Porcentaje de } \\
\text { alumnos que han } \\
\text { respondido } \\
\text { afirmativamente }\end{array}$ \\
\hline Derecho & 58 & $29.6 \%$ & 47 & $81,0 \%$ \\
\hline $\begin{array}{l}\text { Administración y dirección } \\
\text { de empresas }\end{array}$ & 56 & $28,6 \%$ & 47 & $84,0 \%$ \\
\hline $\begin{array}{l}\text { Ciencias del trabajo y } \\
\text { recursos humanos }\end{array}$ & 36 & $18,4 \%$ & 27 & $75,0 \%$ \\
\hline Periodismo & 29 & $14,8 \%$ & 27 & $93,1 \%$ \\
\hline Ingeniería informática & 8 & $4,1 \%$ & 7 & $87,5 \%$ \\
\hline $\begin{array}{l}\text { Empresas y actividades } \\
\text { turísticas }\end{array}$ & 6 & $3,0 \%$ & 5 & $83,3 \%$ \\
\hline Psicología & 2 & $1,0 \%$ & 1 & $50,0 \%$ \\
\hline $\begin{array}{l}\text { Magisterio en educación } \\
\text { primaria }\end{array}$ & 1 & $0,5 \%$ & 0 & $0,0 \%$ \\
\hline Total & 196 & $100 \%$ & 161 & $82,1 \%$ \\
\hline
\end{tabular}

El grado de Periodismo aparece en segunda posición con una puntuación por debajo del valor 2, siendo en este aspecto el único grado junto al de Magisterio en educación primaria, lo que supone que esta propuesta docente se adecua incluso mejor en esta titulación que en otras más relacionados con el entorno empresarial. Este hecho puede ser debido a que la formación recibida ayuda a los estudiantes a organizar mejor su emprendimiento y a conocer más claramente los modelos de negocio a aplicar.

Los alumnos de otros grados que también muestran buenas puntuaciones en este aspecto como son los de Ciencias del trabajo y recursos humanos, Ingeniería informática, Derecho y Administración y dirección de empresas conocen perfectamente los modelos de negocio de las empresas que pueden crear (asesorías, gestorías administrativas o empresas de desarrollo de software, por ejemplo), por lo que este nuevo conocimiento que reciben los futuros periodistas, precisamente en una época en la que su sector tiene una necesidad urgente de reinventar sus modelos de negocio, incrementa la adecuación de la propuesta al fomento del emprendimiento entre los estudiantes de periodismo.

Por lo que respecta a la siguiente pregunta, la consulta a los alumnos ha pretendido reflejar igualmente el grado en que la actividad didáctica ha servido como motivación para el estudio de la asignatura (véase tabla 3), lo que favorece su capacitación para ofrecer en un futuro un servicio de mayor calidad en sus cometidos profesionales. En esta consulta, se ha utilizado la misma escala de Likert y los resultados del ANOVA expuesto en la tabla 3 identifican que igualmente al caso de la pregunta anterior, existen diferencias estadísticamente significativas en la media de los valores entre el grado de periodismo y cada uno de los grados participantes en el estudio.

Igualmente en este caso, la propuesta docente también es adecuada para motivar en el estudio de la asignatura, dado que exceptuándose los estudiantes del grado de Empresas y actividades turísticas, el resto han respondido con puntuaciones muy bajas 
y, por lo tanto, favorables en este sentido. En el caso del grado de Periodismo, sus estudiantes han sido los únicos que han contestado con valores por debajo de 2, por lo que está propuesta ha resultado para ellos muy adecuada de cara a motivarles en el estudio de la asignatura.

Tabla 2. Nivel de adecuación de la propuesta docente principal en el fomento del espíritu emprendedor

\begin{tabular}{|c|c|c|c|c|}
\hline \multirow[b]{2}{*}{ Grados } & \multicolumn{4}{|c|}{$\begin{array}{c}\text { ¿Qué te ha parecido la actividad didáctica } \\
\text { principal para fomentar en ti el espíritu } \\
\text { emprendedor? } \\
\text { (Muy adecuada }=1 \text {; Nada adecuada }=7 \text { ) }\end{array}$} \\
\hline & Media & $\begin{array}{l}\text { Desviación } \\
\text { típica }\end{array}$ & $\begin{array}{c}\text { ANOVA } \\
\text { F } \\
\end{array}$ & $\begin{array}{c}\text { ANOVA } \\
\text { probabilidad }\end{array}$ \\
\hline $\begin{array}{l}\text { Administración y } \\
\text { dirección de empresas }\end{array}$ & 2.89 & 1.50 & 14.4 & 0.00 \\
\hline $\begin{array}{l}\text { Ciencias del trabajo y } \\
\text { recursos humanos }\end{array}$ & 2.36 & 1.27 & 4.99 & 0.03 \\
\hline Derecho & 2.46 & 1.20 & 8.06 & 0.00 \\
\hline $\begin{array}{l}\text { Empresas y actividades } \\
\text { turísticas }\end{array}$ & 3.33 & 2.07 & 10.53 & 0.00 \\
\hline Ingeniería informática & 2.38 & 1.51 & 2.51 & 0.12 \\
\hline $\begin{array}{l}\text { Magisterio en educación } \\
\text { primaria }\end{array}$ & 1.00 & 0.00 & 0.9 & 0.35 \\
\hline Periodismo & 1.76 & 0.79 & --- & $\begin{array}{ll}-- \\
\end{array}$ \\
\hline Psicología & 3.50 & 0.71 & 9.24 & 0.00 \\
\hline
\end{tabular}

Tabla 3. Nivel de adecuación de la propuesta docente principal en la motivación para el estudio de la asignatura

\begin{tabular}{|c|c|c|c|c|}
\hline \multirow[b]{2}{*}{ Grados } & \multicolumn{4}{|c|}{$\begin{array}{l}\text { Qué te ha parecido la actividad didáctica principal } \\
\text { para motivarte en el estudio de la asignatura? (Muy } \\
\text { adecuada }=1 ; \text { Nada adecuada }=7 \text { ) }\end{array}$} \\
\hline & Media & $\begin{array}{l}\text { Desviación } \\
\text { típica }\end{array}$ & $\begin{array}{l}\text { ANOVA } \\
\text { F }\end{array}$ & $\begin{array}{c}\text { ANOVA } \\
\text { probabilidad }\end{array}$ \\
\hline $\begin{array}{l}\text { Administración y } \\
\text { dirección de empresas }\end{array}$ & 2.73 & 1.68 & 9.91 & 0.00 \\
\hline $\begin{array}{l}\text { Ciencias del trabajo y } \\
\text { recursos humanos }\end{array}$ & 2.11 & 1.17 & 2.82 & 0.10 \\
\hline Derecho & 2.65 & 1.33 & 12.90 & 0.00 \\
\hline $\begin{array}{l}\text { Empresas y actividades } \\
\text { turísticas }\end{array}$ & 3.17 & 2.14 & 9.17 & 0.00 \\
\hline Ingeniería informática & 2.63 & 1.92 & 4.56 & 0.04 \\
\hline $\begin{array}{l}\text { Magisterio en } \\
\text { educación primaria }\end{array}$ & 2.00 & 0.00 & 0.16 & 0.69 \\
\hline Periodismo & 1.69 & 0.76 & --- & --- \\
\hline Psicología & 2.50 & 0.71 & 2.13 & 0.15 \\
\hline
\end{tabular}

El motivo de esta favorable adecuación puede encontrarse en el que el ajuste a la realidad y la vocación de servicio que implica la actividad permite adquirir a los estudiantes de este grado conceptos nuevos de aplicación clara y necesaria si pretenden ejercer como periodistas. De esta manera, los conceptos que al comienzo del estudio 
de las unidades didácticas podrían parecer abstractos, se concretan con la realización de la actividad, lo que clarifica la nueva temática y motiva en su estudio. La formación conseguida de esta manera, supone una mayor capacitación en los estudiantes, que podrán ofrecer con los nuevos conocimientos adquiridos una mayor calidad en la prestación del servicio periodístico.

\section{Conclusiones}

A pesar del momento crítico que está viviendo el sector periodístico, está profesión todavía puede ofrecer una prestigiosa carrera a sus futuros graduados basándose en las nuevas oportunidades y formas de hacer periodismo que surgirán y en las que la calidad en la prestación del servicio periodístico será pieza fundamental. Para aprovechar estas nuevas coyunturas, la integración del emprendimiento en la formación de los periodistas, puede resultar básica de cara a que la innovación y la creatividad impulsen el autoempleo o nuevas iniciativas dentro de las empresas periodísticas existentes.

Con la finalidad de que esta formación sea más efectiva, es necesario aportar propuestas que estimulen el espíritu emprendedor y que motiven al alumno a capacitarse para ofrecer una mayor calidad en el servicio que ofrecerá al consumidor. Por todo ello, en este trabajo se expone una propuesta docente que aplica una metodología de aprendizaje de servicio utilizada con éxito por Casero-Ripollés y Cullel-March (2013: 687) para educar en emprendimiento a los graduados en periodismo. Con dicha metodología se pretende que los alumnos generen proyectos con una clara vocación de transformación social y económica, que les permita comprender mejor la realidad con la que tendrán que interactuar en sus cometidos profesionales.

Los resultados de esta propuesta que, ha sido empleada también en otras aulas distintas al Grado de Periodismo, confirman los obtenidos por Casero-Ripollés y CullelMarch (2013: 688). En concreto, partiendo de una muestra de estudiantes que, tras recibir la formación se encuentran en su gran mayoría dispuestos a emprender, y dentro de ellos especialmente los periodistas, el nivel de adecuación de la propuesta para estimular el espíritu emprendedor es elevado, excepto en dos grados que presentan una valoración neutra (Psicología y de Empresas y actividades turísticas). Idéntica calificación se ha obtenido en relación con el nivel de adecuación de la propuesta para motivar en el estudio de la asignatura, con un único grado que ha presentado una valoración neutra (Empresas y actividades turísticas).

En el caso de los alumnos de periodismo, los análisis realizados muestran que han sido los únicos estudiantes que han contestado con valoraciones próximas al nivel de máxima adecuación, por lo que se puede afirmar que esta propuesta docente basada en la metodología de servicio estimula el espíritu emprendedor y la motivación por estudiar de los futuros graduados, y muy especialmente, en los periodistas.

Por todo lo expuesto anteriormente, la experiencia que se ha expuesto corrobora la hipótesis de que las propuestas docentes basadas en la metodología de aprendizaje de servicio fomentan el espíritu emprendedor y la capacitación de los futuros periodistas, por lo que resultan muy propicias para que ellos encuentren nuevas formas de desarrollar su profesión y de engrandecerla en la importante función de divulgación e interpretación de la información que desarrollan en beneficio de toda la sociedad. 


\section{Referencias bibliográficas}

CAMPOS-FREIRE, Francisco (2010): "Los nuevos modelos de gestión de las empresas mediáticas". Estudios sobre el mensaje periodístico, $\mathrm{n}^{\circ} 16$. Madrid, Servicio de Publicaciones de la Editorial Complutense, pp. 13-30.

CASERO-RIPOLLES, Andreu y CULLEL-MARCH, Cristina (2013): "Periodismo emprendedor. Estrategias para incentivar el autoempleo periodístico como modelo de negocio". Estudios sobre el Mensaje Periodístico, n 19. Madrid, Servicio de Publicaciones de la Editorial Complutense, pp. 681-690. http://dx.doi.org/10.5209 /rev_ESMP.2013.v19.42151

CASERO-RIPOLLÉS, Andreu (2012): “Contenidos periodísticos y nuevos modelos de negocio: evaluación de servicios digitales". El profesional de la información, $\mathrm{n}^{\mathrm{o}}$ 21 (4), pp. 341-346. http://dx.doi.org/10.3145/epi.2012.jul.02

CASERO-RIPOLLES, Andreu (2010): "Prensa en internet: nuevos modelos de negocio en el escenario de la convergencia". El profesional de la información, $\mathrm{n}^{\circ} 19$ (6), pp. 595-601. http://dx.doi.org/10.3145/epi.2010.nov05

EDWARDS, Vanessa (2013): "Journalism as a Profession: Careers and Expectsations", en FOWLER-WATT, Karen y ALLAN, Suart: Journalism: New Gallenges. Bournemouth, Centre for Journalism \& Communication Research Bournemouth University, pp. 323-337.

FERNÁNDEZ-LAVIADA, Ana; RUEDA-SAMPEDRO, María Inés; y HERREROCRESPO, Ángel (2011): "Estudio de la actitud emprendedora de los estudiantes universitarios de la facultad de CC. EE. y empresariales de Cantabria”, en XVI Congreso de la Asociación Española de Contabilidad y Administración de Empresas (AECA): http://www.aeca.es/pub/on_line/comunicaciones_xvicongresoaeca/cd/36f.pdf [Consulta: 16 de noviembre de 2013].

FLEW, Ferry (2012): “The digital transformation 21st Century news journalism” en Conference on Digital Media and Journalism Ming Chuan University: http://www.academia.edu/2045520/THE_DIGITAL_TRANSFORMATION_OF_2 1ST_CENTURY_NEWS_JOURNALISM [Consulta: 20 de octubre de 2013].

GARCÍA-LÓPEZ, Javier; MARTÍNEZ-PASTOR, Esther (2013). "Investigación sobre publicidad digital en revistas científicas españolas". El profesional de la información, $\mathrm{n}^{\circ} 22$ (2), pp. 173-180. http://dx.doi.org/10.3145/epi.2013.mar.12

GOÑI-ALEGRE, Belén (2013): "El emprendimiento, responsabilidad de todos". Revista de la $A E C A, \mathrm{n}^{\circ} 103$, pp. 35-36.

GUALLAR, Javier (2010): “Prensa digital en 2009”. Anuario ThinkEPI, n 4, pp. 165 173.

JIMÉNEZ-MORENO, Juan y LEGATO, Ana (2010): "Intención emprendedora en la población juvenil universitaria femenina: estudio preliminar de un caso", en XII Reunión de Economía Mundial: https:/www.lugo.usc.es/congresos/xiirem /pdf/104.pdf [Consulta: 10 de octubre de 2013]. 
JONES, Daniel (2005): “Aproximación teórica a la Estructura de la Comunicación Social”. Sphera Publica: Revista de Ciencias Sociales y de la Comunicación, $\mathrm{n}^{\circ}$ 5, pp. 19-39.

KRUEGER, N. F. y BRAZEAL, D. V. (1994): "Entrepreneurial Potential and Potential Entrepreneurs". Entrepreurship Theory and Practice, no 18 (3), pp. 91-104.

LARRAÑAGA-RUBIO, Julio (2010): "Industria de los periódicos: nuevos modelos económicos y nuevos soportes". Estudios sobre el mensaje periodístico, $\mathrm{n}^{\circ} 16$. Madrid, Servicio de Publicaciones de la Editorial Complutense, pp. 59-78.

LARRAÑAGA-RUBIO, Julio (2009): "La crisis del modelo económico de la industria de los periódicos". Estudios sobre el mensaje periodístico, $\mathrm{n}^{\circ} 15$. Madrid, Servicio de Publicaciones de la Editorial Complutense, pp. 61-80.

LIÑÁN, Francisco y CHEN, Yi-Wen (2009): "Development and Cross-Cultural Application of a Specific Instrument to Measure Entrepreneurial Intentions". Entrepreurship Theory and Practice, $\mathrm{n}^{\circ} 33$ (3), pp. 593-617.

MARINA, José Antonio (2010): "La competencia de emprender". Revista de Educación, $\mathrm{n}^{\circ}$ 351, pp. 49-71.

MARTÍNEZ, Miquel (2008): Aprendizaje de Servicio y Responsabilidad Social de las Universidades. Madrid, Ediciones Octaedro.

MEYER, Philip (1995): "Learning to love Profits" en American Journalism Review (AJR): http://ajrarchive.org/article.asp?id=1461 [Consulta: 20 de octubre de 2013].

ORTEGA, Iñaki (2012): "Política pública de promoción de la capacidad emprendedora en España". Economistas, n ${ }^{\circ}$ 132, pp. 66-71.

OSTERWALDER, Alexander y PIGNEUR, Ives (2011): Generación de modelos de negocio. Barcelona, Deusto Grupo Planeta.

PALACIOS-LLANOS, Luis (2012): Informe Anual de la Profesión Periodística 2012, http://www.apmadrid.es/images/stories/INFORME\%20ANUAL \%20DE\%20LA\% 20PROFESION\%20PERIODISTICA\%202012\%2019,5\%20\%20megas\%20sin\% 20pag_\%20blancas.pdf [Consulta: 12 de octubre de 2013].

PETERMAN, Nicole and KENNEDY, Jessica (2003): "Enterprise Education: Influencing Students' Perceptions of Entrepreurship". Entrepreurship Theory and Practice, $\mathrm{n}^{\circ} 28$ (2), pp. 129-144.

PRICEWATERHOUSECOOPERS (2013): “Global Entertainment and Media Outlook: 2013-2017". https://www.pwc.com/outlook [Consulta: 15 de octubre de 2013].

REAL ACADEMIA ESPAÑOLA (2013): “Emprender”, en: http://lema.rae.es/drae /srv/search?id=F77BO4xYqDXX2BpwCGRr [Consulta: 18 de octubre de 2013].

TOLEDANO-GARRIDO, Nuria (2006): "Las perspectivas empresariales de los estudiantes universitarios". Revista de Educación, n 341, pp. 803-828.

THOMPSON, Shelley (2013): "The future of Newspaper in a Digital Age", in FOWLER-WATT, Karen y ALLAN, Suart: Journalism: New Gallenges. 
Bournemouth, Centre for Journalism \& Communication Research Bournemouth University, pp. 19-34.

THOMPSON, Donald (2012): Oracles: How Prediction Markets Turn Employees into Visionaries. USA, Harvard Business School Publishing.

VON GRAEVENITZ, Georg; HARHOFF, Dietmar; and WEBER, Richard (2010): "The effects of entrepreneurship education". Journal of Economic Behavior \& Organization, $\mathrm{n}^{\mathrm{o}}$ 76, pp. 90-112. http://dx.doi.org/10.1016/j.jebo.2010.02.015 